\title{
Pathologic Stage IB Gastric Cancer AJCC v8
}

National Cancer Institute

\section{Source}

National Cancer Institute. Pathologic Stage IB Gastric Cancer AJCC v8. NCI Thesaurus.

Code C133657.

Stage IB includes: (T1, N1, M0); (T2, N0, M0). T1: Tumor invades the lamina propria, muscularis mucosae, or submucosa. T2: Tumor invades the muscularis propria. N0: No regional lymph node metastasis. N1: Tumor with metastasis in one or two regional lymph nodes. M0: No distant metastasis. (AJCC 8th ed.) 